\title{
Health Condition Assessment of Marine Systems Based on an Improved Radar Chart
}

\author{
Yuewen Zhang $\mathbb{D},{ }^{1}$ Peng Zhang $\left(\mathbb{D},{ }^{1}\right.$ Boshen Zhang $\left(\mathbb{D},{ }^{2}\right.$ and Peiting Sun $\mathbb{D}^{1}$ \\ ${ }^{1}$ Marine Engineering College, Dalian Maritime University, Dalian 116026, China \\ ${ }^{2}$ Australia Maritime College, University of Tasmania, Hobart, Tasmania 7248, Australia \\ Correspondence should be addressed to Peng Zhang; zhangpenglunji@dlmu.edu.cn
}

Received 21 September 2020; Revised 30 October 2020; Accepted 23 November 2020; Published 11 December 2020

Academic Editor: Paolo Crippa

Copyright $\odot 2020$ Yuewen Zhang et al. This is an open access article distributed under the Creative Commons Attribution License, which permits unrestricted use, distribution, and reproduction in any medium, provided the original work is properly cited.

Since health assessment plays a significant role in marine systems (MSs), it has caught the attention of researchers. In this study, a powerful evaluation method called an improved radar chart was developed as a means of reliability estimation. General evaluation methods applied in the comprehensive evaluation of MS are slightly insufficient in terms of considering index coordination. However, the application of a radar chart can solve this problem. To improving the shortcomings of a traditional radar chart, the fuzzy centralization statistical theory and the entropy weight were combined in this study to obtain the comprehensive weight. The weight could be converted into an angle, and it could reflect the influence degree of the indexes on the evaluation objects. Additionally, an angle bisector was introduced as an index axis, and the eigenvector was extracted to get the unique evaluation result. The result showed that the proposed method could achieve the continuous online monitoring of the system state, and the reliable and accurate assessment results were able to provide a reference for condition-based maintenance and decision-making.

\section{Introduction}

With the improvement in ship intellectualization, MSs are becoming more automated and autonomous. Generally speaking, MS can be operated with few or no human operators on board, which may decrease the risk of operation in relation to crew injuries and fatalities [1]. Therefore, it is necessary to determine the performance reliability at all times. Additionally, when facing uncertainty or unanticipated events, MSs also require decision-making, problem solving, and strategy implementation. Accurate state assessment can provide a reference for operation and maintenance as well.

A variety of decision-making problems with aggregation operators can be seen in the reference literature. For example, extended power average operators for decisionmaking were applied to an emergency response plan selection for civil aviation [2], and the geometric Bonferroni mean aggregation operators and an enhanced ordered weighted averaging operators generation algorithm were proposed to solve the multiple criteria decision-making problems [3, 4]. Pythagorean fuzzy sets have been widely used in the expression of the opinions of decision makers for uncertainty. The evaluation of online payment service providers has been provided to illustrate the validity and merits of the Pythagorean fuzzy interaction power Bonferroni mean aggregation operators in multiple attribute decision-making [5]. When the maintenance of MS is performed, many factors need to be considered in making decisions based on health values. Health condition assessment has been based on advanced monitoring methods. In combination with reliable and effective evaluation methods, it could be used to monitor the health status of MS and to judge the development of health conditions. At the same time, according to the results of health assessments and analyses, warnings for possible faults and a basis for assistant 
decision-making could also be provided. Additionally, premaintenance, cost savings, reliable operation, and safety navigation could be achieved. At present, health condition assessment is widely used in lithium batteries, aerospace, bridges, power grids, nuclear power, and other fields [6-9].

Rana et al. [10] assumed that the system state was a set of random variables that were moving between the factor and variable nodes and that had mean and covariance values. Then, according to structure principle of Bayesian filter, the state estimation of the smart grid was achieved by iterating the information repeatedly. To reduce the influence of uncertainties such as model parameters as well as measurement and instrument noise, Afshari et al. [11] proposed a robust state estimation method called the smooth variable structure filter (SVSF). It could improve the performance of the state assessment model at the same time. Zahid et al. [12] proposed a new charge state estimation method that was based on subtractive clustering. The simulation experiments were conducted with an advanced vehicle simulator. The results showed that compared to a backpropagation neural network and an Elman neural network, under different driving cycles, the proposed model exhibited remarkable progress in charge state estimation with high potential. $\mathrm{Xu}$ et al. [13] described a state discretization method to simulate how the health status of one component affected the risk rate of another component. The extended proportional hazard model (PHM) was used to characterize the failure correlation and to estimate the impact of the degradation state of one component on the hazard degree of another component. Kumar et al. [14] discussed a tool health state estimation model that was based on time-series sensor signal sequence clustering. The validity of the method was verified with a numerical control drilling test rig equipped with thrust and torque sensors. At the same time, different evaluation methods were adopted to evaluate the battery state [15-18]. In addition, the data-driven method was used to deduce the health index of the ACS from multisensor signals. After that, a dynamic linear model was proposed to describe the degradation process of the failure prediction. The Bayesian inference formula was used to estimate and predict the degradation [19]. Radar state assessment has been applied in many fields [20-22]. It is suitable for solving the health evaluation problem of multiequipment and multiparameters.

Obviously, different index parameters have different effects on the health status. The health values depend on the degree of the evaluation index, and they are influenced by the correct evaluation of the relative importance of the index. At present, there are two kinds of commonly used methods for determining the weight of each index: subjective methods and objective methods. In a comprehensive evaluation, to improve the rationality of the weight value and increase the accuracy of the evaluation results, the combination of these two methods is generally used to obtain the combination weight. The Pythagorean fuzzy interactive Hamacher power aggregation operators with entropy weight were proposed for assessment of express service quality [23]. Ghosh et al. [24] used a genetic algorithm to learn the optimal fusion weight through a correlation evaluation and a method for attribute weight estimation based on a rough set that had been presented [25]. A multiattribute comprehensive evaluation method for power transmission and transformation engineering was proposed [26].

MSs are usually composed of many kinds of subsystems or equipment types. Each subsystem or type of equipment is independent of each other, and it has internal relationships. Additionally, the subsystems and equipment in a system are often designed to accomplish specified tasks. Thus, their physical functions are different, and the number of monitoring parameters collected by sensors is large. Additionally, a ship system has the characteristics of multiparameters and multiequipment. This makes the health assessment of a ship system very complex.

The objective of this research was to propose a method to solve the problem of MS health condition assessment. The proposed indexes were evaluated for applicability in operational decision-making and performance monitoring at first. Additionally, for the problems of determining the weight of evaluation index and establishing an evaluation model, based on the principle of minimizing the total evaluation value, the fuzzy centralization statistical theory (FCST) and the entropy weight were applied to confirm the combination weight. Then, according to the changes in the data, a dimensionless method of monitoring data was proposed. Finally, an evaluation model was established by using the improved radar chart (IRC) method.

Considering the problems and shortcomings of traditional methods, a health condition assessment model based on an improved radar chart was proposed. The main contributions of this paper can be summarized as follows:

(i) An indicator system for condition assessment based on the system function analysis method was proposed. Considering unmanned ships, this method could better reflect the technical status of MS.

(ii) An IRC model for health condition assessment was creatively developed. The FCST and the entropy weight were applied in the model to confirm the combination weight.

(iii) The proposed method achieved continuous online assessment, which made it possible to detect potential faults and to exhibit a potential capability in tracing the further deterioration of the system.

The structure of this article is given below. Section 2 describes the framework of the condition assessment. In Section 3, a brief introduction of evaluation index is given. The proposed IRC method is described in Section 4. Section 5 describes how the fuel oil supply system was chosen as an example to verify the feasibility of the method. The conclusions are given in Section 6.

\section{Framework of the Condition Assessment}

To study the real-time health condition assessment, in this research, a framework based on a dynamic health assessment model was proposed. Figure 1 shows the data flow in the proposed framework. The proposed real-time condition 


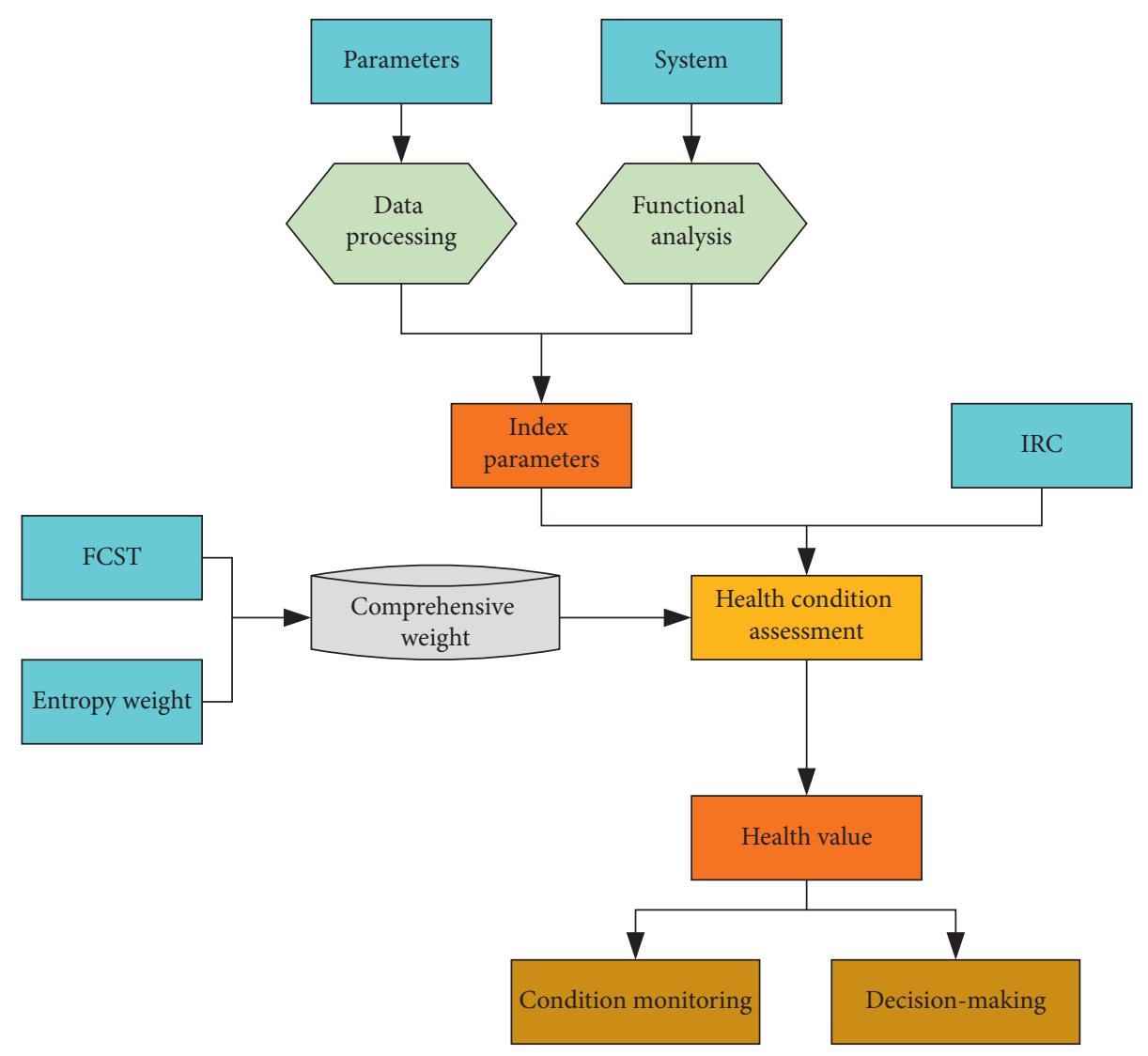

FiguRE 1: Framework of the condition assessment.

assessment was constructed based on the IRC model. Seven steps were used to calculate the health values. The detailed processes of each step are introduced as follows:

(i) Step 1: the assessment indicator system was established via functional analysis. Through the analysis of the system, the parameters that could best represent the change of the system were selected as the assessment index.

(ii) Step 2: the system operation data under normal conditions were collected from the database, and the raw data were processed.

(iii) Step 3: the weight of the indexes was calculated with the comprehensive weight method.

(iv) Step 4: the IRC model was established based on the radar chart and the comprehensive weight.

(v) Step 5: the dynamic health assessment model was built based on Steps 1-4.

(vi) Step 6: the model was applied to the selected system, and the health value was calculated.

(vii) Step 7: the condition monitoring was achieved, and the operation and maintenance decisions were made according to the health value.

In this research, the proposed method was presented to solve those questions and to produce the systems dynamic reliability assessment. In the case study described in Section
5, the system was considered a series system for which the abnormality of one component could result in the changes in the health status of the system.

\section{Index System of Comprehensive Evaluation}

To realize the state evaluation, it was necessary to select the corresponding index according to the specific functional requirements. MSs are more concerned with whether the systems and equipment can meet the voyage requirements (port maintenance mode); that is, whether the technical status can guarantee the minimum functional requirements in the voyage process. As described in this section, first, through the system function analysis, the suitable index parameter was selected. Second, the parameterization was conducted for each index. Finally, the weight of the index was calculated.

3.1. Determination of System Evaluation Index. To be able to identify all potential failures, a reliability engineer has to have a thorough understanding of the various functions. A functional analysis is therefore an important step in a system reliability analysis. A complex system may have a high number of required functions. All functions are however, not equally important, and a classification may therefore be an aid for identification and analysis purposes. One way to classify functions is essential functions, auxiliary functions, 
protective functions, safety functions, hygiene functions, information functions, interface functions, and superfluous functions [27]. The steps to determine the indicator system were as follows:

(i) Step 1: defining functions. The functions of the systems and equipment were described generally.

(ii) Step 2: functional analysis. According to the relationship, the functions were divided into main functions, auxiliary functions and additional functions.

(iii) Step 3: functional arrangement. The functions were arranged according to logical relationships. Upper functions were on the left, and lower functions were on the right. First, the main functions were arranged, and then, the auxiliary functions were arranged.

(iv) Step 4: determining the indicator parameters. By combining the function model diagram with the specific process of realizing the function, the most critical monitoring parameters in the physical process of realizing the main function were determined.

\subsection{Index Weight Calculation}

3.2.1. Subjective Weight. All the artificially determined index weights were uncertain and random. If a specific value was used to measure the weight of the indexes, the accuracy of the results would be reduced. In contrast, the uncertainty and the randomness that existed in the weights of the

$$
\begin{gathered}
\bar{A}\left(u_{i}\right)= \\
\left(a_{r i}, b_{r i}\right)= \\
\\
\bar{A}\left(u_{i}\right) \mathrm{d} u \\
\bar{A}\left(u_{i}\right) \mathrm{d} u
\end{gathered}
$$

By simplifying, the following equation is obtained:

$$
\bar{\psi}_{i}^{\prime}=\frac{1 / 2 \sum_{r=1}^{q}\left(b_{r i}^{2}-a_{r i}^{2}\right)}{\sum_{r=1}^{q}\left(b_{r i}-a_{r i}\right)} .
$$

At this point, the relative weight $\vec{\psi}_{i}$ of the evaluation index $u_{i}$ was

$$
\begin{gathered}
\bar{\psi}_{i}^{\prime}=\frac{\int_{u_{i \min }}^{u_{i \max }} u_{i} \bar{A}\left(u_{i}\right) \mathrm{d} u}{\int_{u_{i \min }}^{u_{i \max }} \bar{A}\left(u_{i}\right) \mathrm{d} u}, \\
u_{i \min }=\min \left(a_{1 i}, a_{2 i}, \ldots, a_{q i}\right), \\
u_{i \max }=\max \left(a_{1 i}, a_{2 i}, \ldots, a_{q i}\right) .
\end{gathered}
$$

TABLE 1: Expert score interval.

\begin{tabular}{lcccccc}
\hline $\begin{array}{l}\text { Evaluation } \\
\text { expert }\end{array}$ & $u_{1}$ & $u_{2}$ & $\ldots$ & $u_{i}$ & $\ldots$ & $u_{m}$ \\
\hline$p_{1}$ & {$\left[a_{11}, b_{11}\right]$} & {$\left[a_{12}, b_{12}\right]$} & $\ldots$ & {$\left[a_{1 i}, b_{1 i}\right]$} & $\ldots$ & {$\left[a_{1 m}, b_{1 m}\right]$} \\
$p_{2}$ & {$\left[a_{21}, b_{21}\right]$} & {$\left[a_{22}, b_{22}\right]$} & $\ldots$ & {$\left[a_{2 i}, b_{2 i}\right]$} & $\ldots$ & {$\left[a_{2 m}, b_{2 m}\right]$} \\
$\ldots$ & $\ldots$ & $\ldots$ & $\ldots$ & $\ldots$ & $\ldots$ & $\ldots$ \\
$p_{r}$ & {$\left[a_{r 1}, b_{r 1}\right]$} & {$\left[a_{r 2}, b_{r 2}\right]$} & $\ldots$ & {$\left[a_{r i}, b_{r i}\right]$} & $\ldots$ & {$\left[a_{r m}, b_{r m}\right]$} \\
$\ldots$ & $\ldots$ & $\ldots$ & $\ldots$ & $\ldots$ & $\ldots$ & $\ldots$ \\
$p_{q}$ & {$\left[a_{q 1}, b_{q 1}\right]$} & {$\left[a_{q 2}, b_{q 2}\right]$} & $\ldots$ & {$\left[a_{q i}, b_{q i}\right]$} & $\ldots$ & {$\left[a_{q m}, b_{q m}\right]$} \\
\hline
\end{tabular}

artificial evaluation indexes were taken into account, and a range interval was used to replace the specific value. The index weight determined in this way would have a higher accuracy and be more in line with the actual situation. FCST was a subjective weighting method that considered uncertainty and randomness.

The objective weight determination process was as follows: an evaluation system was set up with $m$ indexes, which could be noted as $U=\left\{u_{1}, u_{2}, \ldots, u_{i}, \ldots, u_{m}\right\}$. The number of relevant experts required to determine the weight value of indexes was $q$, and the set was $P=\left\{p_{1}, p_{2}, \ldots, p_{r}, \ldots, p_{q}\right\}$. An eigenvalue interval $\left[a_{r i}, b_{r i}\right] \quad(i=1,2, \ldots, m$, $r=1,2, \ldots, q)$ was given by the evaluation expert $P$ according to his or her evaluation criteria and understanding of the relevant conditions of each index. The corresponding set value interval of each indicator is shown in Table 1.

For the indexes, the set value interval given by the experts was combined to obtain the set value statistical sequence. The sample drop shadow function $\bar{A}\left(u_{i}\right)$ was used to represent the distribution of the sequence after stacking:
Owing to the different knowledge areas, experience, and opinions of the experts, the value interval of the weight set given by the experts was different. Therefore, it was necessary to determine the weight interval given by the experts; that is, the importance degree of the expert opinions $\sum_{r=1}^{q} k_{r}=1$.

To make the obtained index weight comprehensive, three levels could be selected for consultation: management engineer of a shipping company, manager-level engineer on a ship, and support-level engineer. Because the position reflected the level of knowledge and the experience of the expert, the importance of each expert's opinion was determined according to the position level. The expert positions involved include chief engineer, second engineer, third engineer, and fourth engineer. The expert opinions were graded according to the corresponding positions, as shown in Table 2. 
TABLE 2: Grading and scoring for expert opinions.

\begin{tabular}{lccc}
\hline Level of opinion & Level 1 & Level 2 & Level 3 \\
\hline Mark of opinion & 3 & 2 & 1 \\
Specialist position & Engineer, chief engineer & Second engineer & Third engineer, fourth engineer \\
\hline
\end{tabular}
follows.

The classification of the expert opinions is described as

Among all three levels, the chief engineers had the highest level of experience and knowledge in managing the machinery and equipment of each ship. As a result, they were all classified as level 1. According to their knowledge and experience, the second engineers were classified into the second grade. The third and fourth engineers had a relative lack of management experience and knowledge, so their experience levels were relatively low. However, considering their knowledge of ship machinery and equipment, they were also consulted and classified as level 3 .

Thus, as shown in equation (4), the weight of each expert opinion could be obtained by normalizing the expert opinion score:

$$
k_{i}=\frac{s_{i}}{\sum_{i=1}^{q} s_{i}},
$$

where $k_{i}$ is the weight value of expert opinion $i, s_{i}$ is the score of expert opinion $i$, and $q$ is the number of experts.

After considering the importance of the weight interval that was given by the experts, the relative weight of the indexes could be calculated with the following equation:

$$
\bar{\psi}_{i}^{\prime}=\frac{1 / 2 \sum_{r=1}^{q} k_{r}\left(b_{r i}^{2}-a_{r i}^{2}\right)}{\sum_{r=1}^{q} k_{r}\left(b_{r i}-a_{r i}\right)} .
$$

The subjective weight of each of the indexes could be obtained by calculation according to the following equation: $\psi_{i}=\bar{\psi}_{i}^{\prime} / \sum_{i=1}^{m} \vec{\psi}_{i}^{\prime}$.

Finally, the corresponding subjective weight vector of the health status assessment index system was $\left(\psi_{1}, \psi_{2}, \ldots, \psi_{m}\right)$.

According to the basic principle of FSC, the uncertainty of an expert opinion increased with the weight setting interval. According to this characteristic, the reliability analysis of the calculated subjective weight vector could be carried out.

(1) Confidence method. For the index $u_{i}$, the confidence of its weight was defined as

$$
d_{i}=1-\frac{1}{q} \sum_{r=1}^{q}\left[b_{r i}-a_{r i}\right], \quad 0 \leq d_{i} \leq 1 .
$$

(2) Interval variance method. For the index $u_{i}$, the variance $F_{i}$ was used to measure reliability:

$$
F_{i}=\frac{\sum_{r=1}^{q}\left[\left(b_{r i}-\bar{\psi}_{i}\right)^{3}-\left(a_{r i}-\bar{\psi}_{i}\right)^{3}\right]}{3 \sum_{r=1}^{q}\left(b_{r i}-a_{r i}\right)} .
$$

3.2.2. Objective Weight. The objective weight could make full use of the internal correlation of the monitoring data. The most representative method was the entropy weight method [28]. Entropy is a measure of the uncertainty degree of system information, which provides a new theoretical basis for the determination of objective weight. Therefore, an entropy weight method was developed to objectively assign weights according to the amount of information transmitted by each evaluation index. The weighted results could reflect the degree of disorder of the monitoring information.

It was assumed that the number of indexes of evaluation objects was $n$ and that each indicator had monitoring data with a number of $m$ groups. These monitoring data constituted the matrix $X$ :

$$
X=\left(x_{i j}\right)_{m \times n}=\left[\begin{array}{cccc}
x_{11} & x_{12} & \ldots & x_{1 n} \\
x_{21} & x_{22} & \ldots & x_{2 n} \\
\vdots & \vdots & \ddots & \vdots \\
x_{m 1} & x_{m 2} & \ldots & x_{m n}
\end{array}\right] .
$$

The steps taken to determine the objective weight vector of evaluation index system are shown:

(1) The monitoring data were processed with $P_{i j}=x_{i j} / \sum_{i=1}^{m} x_{i j}$.

(2) The information entropy $E_{j}$ of the jth evaluation index $E_{j}=-1 / \ln n \cdot \sum_{i=1}^{n}\left(p_{i j} \ln p_{i j}\right),(0 \ln 0=0)$ was calculated.

(3) The index entropy weight $\varphi_{j}$ was determined:

$$
\begin{aligned}
\varphi_{j} & =\frac{1-E_{j}}{n-\sum_{j=1}^{m} E_{j}}, \quad \varphi_{j} \in[0,1], \\
\sum_{j=1}^{m} \varphi_{j} & =1 .
\end{aligned}
$$

In conclusion, the objective weight vectors corresponding to the evaluation index system were calculated as $\left[\varphi_{1}, \varphi_{2}, \ldots, \varphi_{n}\right]$.

3.2.3. Comprehensive Weight. To minimize the deviation of the subjective and objective weights, a comprehensive weight was constructed based on optimization theory. Since the monitoring parameters were dimensionless, it was known that the health assessment system in the jth parts of the subjective and objective weights of the evaluation indexes were bits of $\psi_{i}$ and $\varphi_{j}$, respectively. Letting the 
optimized combination weight be $\omega_{j}$, the corresponding evaluation value $f_{i}$ of the monitoring data of group $i$ was $f_{i}=\sum_{j=1}^{m} \omega_{j} x_{i j}^{\prime}$.

For all the objects to be evaluated in the system, the smaller the total deviation of the evaluation value $F(\omega)$ was, the better the effect was. Therefore, the nonlinear programming model of the total deviation minimization of the evaluation values was established. By solving the model, the optimal combination weight value of evaluation index could be noted as

$$
\begin{cases}\min & F(\omega)=\sum_{i=1}^{m} \sum_{j=1}^{n}\left(\left[\left(\psi_{j}-\omega_{j}\right) x_{i j}^{\prime}\right]^{2}+\left[\left(\varphi_{j}-\omega_{j}\right) x_{i j}^{\prime}\right]^{2}\right), \\ \text { s.t. } & \sum_{j=1}^{n} \omega_{j}=1, \omega_{j} \geq 1 .\end{cases}
$$

\section{Improved Radar Chart}

In a traditional radar map, the length of the number line represents the index value, and the number line is uniformly distributed without consideration of the influence of index weight. Based on this, the index weight of the improved radar map (Figure 2) was represented by the circular angle of the sector where the index line was located. This method highlighted the influence of the index weight in comprehensive evaluation and made the analysis more scientific and effective. In addition, when the improved radar map method was used to conduct the comprehensive assessment of a multi-index parameter system, the radar map of the assessment objects was usually drawn according to the quantity, value, and weight of the evaluation indexes. The characteristic vectors (area and perimeter) of the radar map could be extracted, and the evaluation function could be constructed for quantitative analysis. In the quantitative analysis, the area reflected the overall state of the evaluation object, and the perimeter represented the equilibrium of the development of each index. This meant that when the area was constant and the circumference was smaller, the radar pattern was more approximate to a circle and the values of each index tended to be equal and balanced.

The steps for the health status assessment using the radar map were as follows:

(1) Data Standardization. The initial matrix $X=\left(x_{i j}\right)_{m \times n}$ was dimensionless, and a new matrix $X^{\prime}=\left(x_{i j}^{\prime}\right)_{m \times n}$ was obtained, where $m$ is the number of objects and $n$ is the number of indexes.

(2) Draw Radar chart. First, with point 0 as the center, according to the number of evaluation indexes, $n$ index lines were drawn. Then, according to the index weight, the circular angle of the sector where the

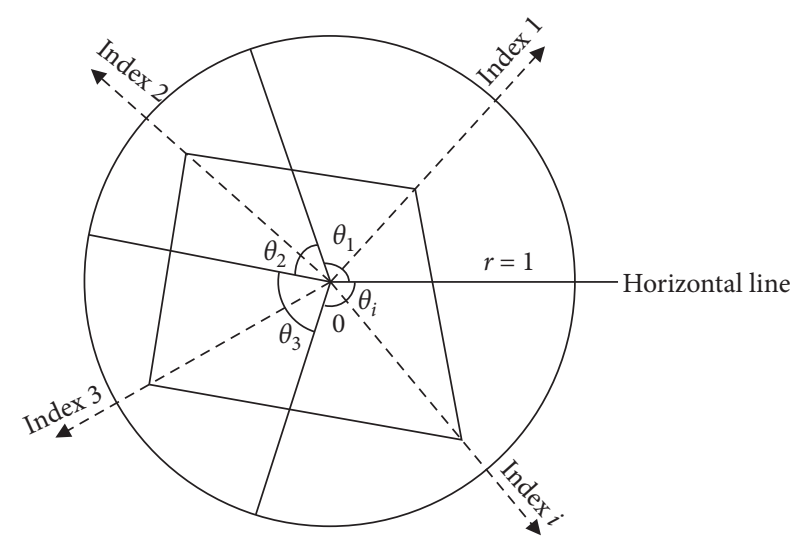

FIgURE 2: Improved radar method sketch map.

index line was located was determined, where $\theta_{i j}=$ $2 \pi \omega_{i j}$ and $\omega_{i j}$ is the weight of the index. Finally, the angle bisector of the circular angle was taken as the index line and the index value was marked on the corresponding index line. Then the marker points were connected on the adjacent index line with a line segment. The irregular polygon that was formed was an improved radar map with index weight.

(3) Extraction Feature Vector. Letting the area of the radar map of the $i$ th evaluation object be $S_{i}$, the perimeter be $L_{i}$, the index number be $j$, and the standard value of the $j$ th index be $x_{i j}^{\prime}$; according to the positive and cosine theorems of trigonometric functions, the area and the perimeter of the radar graph could be obtained:

$$
\begin{aligned}
S_{i} & =\sum_{j=1}^{n} \frac{1}{2} x_{i j}^{\prime} x_{i(j+1)}^{\prime} \sin \theta, \\
L_{i} & =\sum_{j=1}^{n} \sqrt{\left(x_{i j}^{\prime}\right)^{2}+\left(x_{i(j+1)}^{\prime}\right)^{2}-2 x_{i j}^{\prime} x_{i(j+1)}^{\prime} \cos \theta}, \\
\theta & =\frac{\theta_{i j}+\theta_{i(j+1)}}{2} .
\end{aligned}
$$

(4) Construct Evaluation Function. The evaluation vector $V_{i}=\left[v_{i 1}, v_{i 2}\right]$ was defined, with $v_{i 1}=S_{i} / S_{m}$ and $v_{i 2}=S_{i} / \pi \times\left(L_{i} / 2 \pi\right)^{2}=4 \pi S_{i} / L_{i}^{2}$.

$S_{m}$ represents the area value when each index reached the ideal value. The vector component $v_{i 1}$ is the area evaluation value whose magnitude reflected the overall level of the evaluation object. $v_{i 2}$ is the evaluation value of the perimeter, which was the area ratio of the same perimeter, reflecting the equilibrium degree of the evaluation index. It could be found 
that the evaluation vector was comprehensively considered based on two aspects: the overall level of the index and the degree of equilibrium.

Since the evaluation vector was a two-dimensional vector, the geometric average could be used as its evaluation function:

$$
f_{i}=\sqrt{v_{i 1} v_{i 2}}
$$

\section{Case Study}

In the case study, a main engine fuel oil supply system was selected as the research object, and the feasibility of the method was verified. All of the data described below were from the real ship operation parameters. The function of the system was to provide suitable fuel for combustion. If the system failed unexpectedly, the marine propulsion system would shut down, and this would directly affect the safety of the ship. The system was composed of many components, i.e., the duplex filter (DF), supplying pump (SP), circulating pump (CP), autofilter (AF), atomizing heater (AH), oil mixing box, pipeline, and accessories. The structure of the system is shown in Figure 3.

5.1. Index Selection and Parameterization. This research only examined the health assessment of the system under heavy oil conditions, so the effect of oil conversion was not considered:

(1) Fuel heating was achieved by the $\mathrm{AH}$ and the automatic control process. The purpose of the automatic control was to keep the fuel viscosity within the set range, so the key parameter was the fuel viscosity $V$ past the heater.

(2) Fuel purification was achieved through the filter under the action of the fuel pressure differential, and its degree of realization affected the pressure difference on both sides of the filter. Therefore, the pressure differentials $\left(\Delta p_{1}\right.$ and $\left.\Delta p_{2}\right)$ between the inlet and the outlet of the DF and AF were selected as the key parameters.

(3) In the fuel pressurization, whether the fuel SP was pressurized or the fuel $\mathrm{CP}$ was pressurized, the purpose was to increase the fuel pressure to a certain value, so the fuel pressures $p_{1}$ and $p_{2}$ at the outlet of the fuel SP and CP were the key parameters. Based on the above analysis, the indicator system of the health assessment was finally established, as shown in Figure 4.

The following five parameters were selected as the health status evaluation indexes. According to the instruction book and the monitoring data, the benchmark data for each index were determined and classified. The details of the indexes are shown in Table 3.

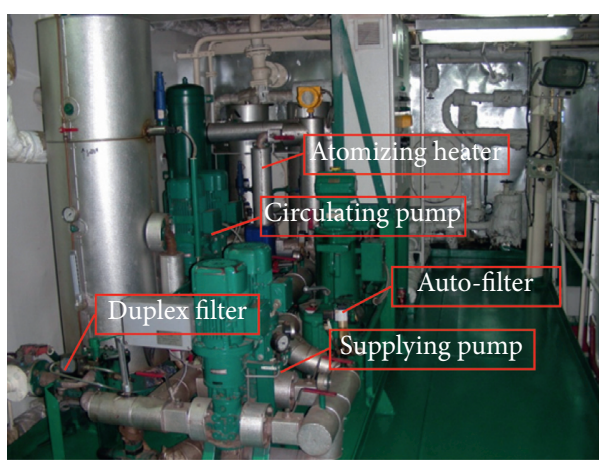

FIgURE 3: The composition of the main engine fuel oil supply system.

5.2. Index Weight Calculation. According to the basic principle of the FCST, eight experts are selected for consultation. The results of the consultation are shown in Table 4.

According to Table 4 and the principles of the FSCT, the weight value of the expert opinions was calculated as $k=[0.07,0.13,0.20,0.13,0.20,0.07,0.13,0.07]$.

The final subjective weight of the evaluation index system of the system was $\psi=[0.10,0.15,0.26,0.25,0.24]$.

Then, the confidence degree and the interval variance of the subjective weight were calculated for the weight confidence of $d=[0.88,0.89,0.90,0.90,0.90]$. This indicated that the expert opinions were relatively uniform, and the results were highly reliable. The interval variance was $F=[0,0,0,0.01,0]$, and its value was small. This indicated that there was little difference in the expert opinions, and the results were highly reliable.

The objective weight of the index according to the entropy weight calculation method is shown in Figure 5. When the monitoring data fluctuated, the state of the corresponding equipment was not stable. Therefore, the corresponding entropy value was high, and the objective weight value was large. As a result, the objective weight value of the corresponding index parameters could be adjusted to make the results more in line with the actual situation.

Finally, combined with the subjective weight, the optimal weight nonlinear programming model was solved based on the minimization principle of the total evaluation estimation deviation and the optimal combined weight value of each evaluation index of the system was obtained. The combined weight vector of each index was about $\omega=[0.05$, $0.15,0.15,0.2,0.45]$.

5.3. Health Assessment of Fuel Oil Supply System. The target ship was a ship for training and scientific research launched in 2008. It usually sailed for short distance navigation, and it seldom encountered severe sea conditions. Therefore, the maintenance of the ship machinery and equipment was good. The monitoring data for each index under heavy fuel oil conditions during January 2019 to July 2019 were extracted. The current data 


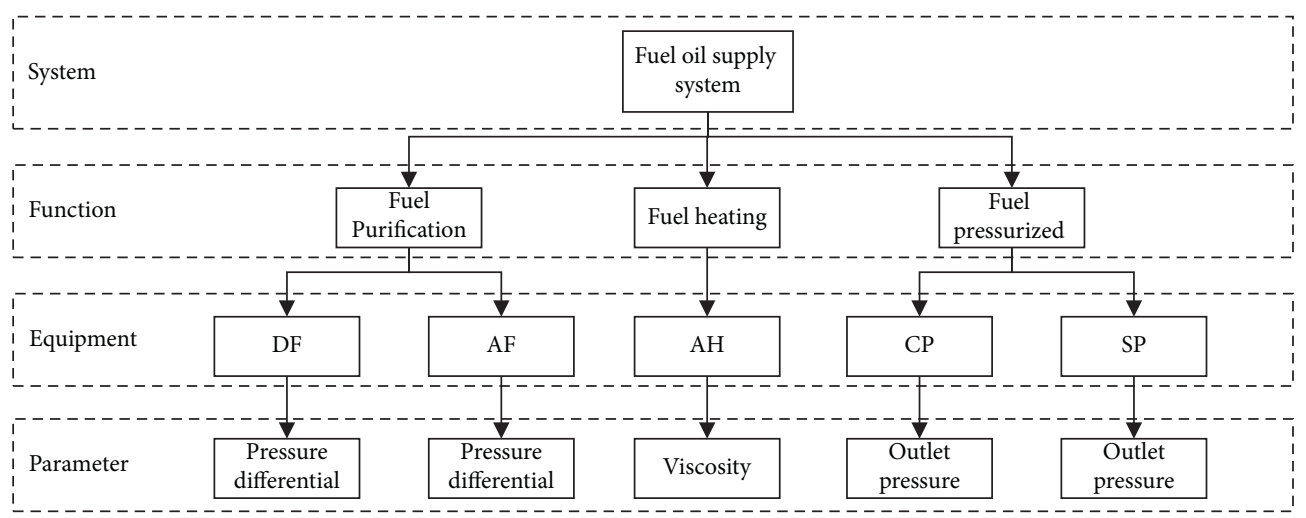

Figure 4: Health assessment indicator system for the fuel oil supply system.

TABLE 3: Benchmarks of health assessment indexes for fuel supply system.

\begin{tabular}{|c|c|c|c|c|c|c|}
\hline \multirow{2}{*}{ Function } & \multirow{2}{*}{ Equipment } & \multirow{2}{*}{ Index } & \multirow{2}{*}{ Unit } & \multicolumn{3}{|c|}{ Benchmark data } \\
\hline & & & & Lower limit & Optimal value & Upper limit \\
\hline \multirow{2}{*}{ Purification } & $\mathrm{DF}$ & $\Delta p_{1}$ & bar & 0.2 & 0.2 & 0.8 \\
\hline & $\mathrm{AF}$ & $\Delta p_{2}$ & bar & 0.2 & 0.3 & 0.8 \\
\hline Heating & $\mathrm{AH}$ & $V$ & $\mathrm{cSt}$ & 7.0 & $12.0 \sim 14.0$ & 20.0 \\
\hline \multirow{2}{*}{ Pressure } & SP & $p_{1}$ & bar & 3.0 & 5.0 & 5.0 \\
\hline & $\mathrm{CP}$ & $p_{2}$ & bar & 6.0 & 8.0 & 11.0 \\
\hline
\end{tabular}

TABLE 4: Weight set value interval of evaluation indexes.

\begin{tabular}{|c|c|c|c|c|c|}
\hline \multirow{2}{*}{ Expert } & \multicolumn{5}{|c|}{ Index system } \\
\hline & $\Delta p_{1}$ & $\Delta p_{2}$ & $V$ & $p_{1}$ & $p_{2}$ \\
\hline$\overline{p_{1}}$ & $10 \sim 20$ & $10 \sim 20$ & $15 \sim 25$ & $20 \sim 30$ & $20 \sim 30$ \\
\hline$p_{2}$ & $5 \sim 15$ & $10 \sim 20$ & $25 \sim 35$ & $10 \sim 20$ & $25 \sim 35$ \\
\hline$p_{3}$ & $5 \sim 15$ & $10 \sim 20$ & $20 \sim 30$ & $30 \sim 40$ & $10 \sim 20$ \\
\hline $\begin{array}{l}P 3 \\
p_{4}\end{array}$ & $1 \sim 10$ & $10 \sim 20$ & $15 \sim 25$ & $20 \sim 35$ & $20 \sim 35$ \\
\hline$p_{5}$ & $5 \sim 18$ & $14 \sim 25$ & $25 \sim 35$ & $15 \sim 28$ & $18 \sim 26$ \\
\hline$p_{6}$ & $20 \sim 30$ & $20 \sim 28$ & $15 \sim 25$ & $13 \sim 20$ & $13 \sim 20$ \\
\hline$p_{7}$ & $1 \sim 10$ & $1 \sim 10$ & $20 \sim 40$ & $20 \sim 40$ & $20 \sim 40$ \\
\hline$p_{8}$ & $12 \sim 17$ & $8 \sim 12$ & $22 \sim 28$ & $18 \sim 22$ & $28 \sim 32$ \\
\hline
\end{tabular}

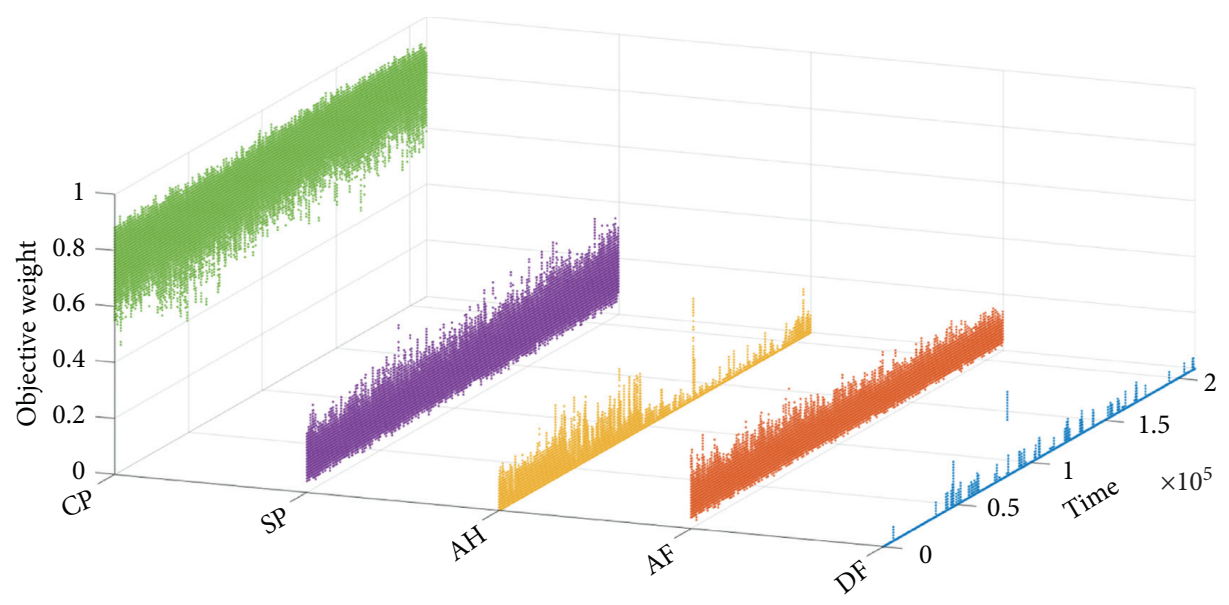

Figure 5: Objective weight of evaluation index. 


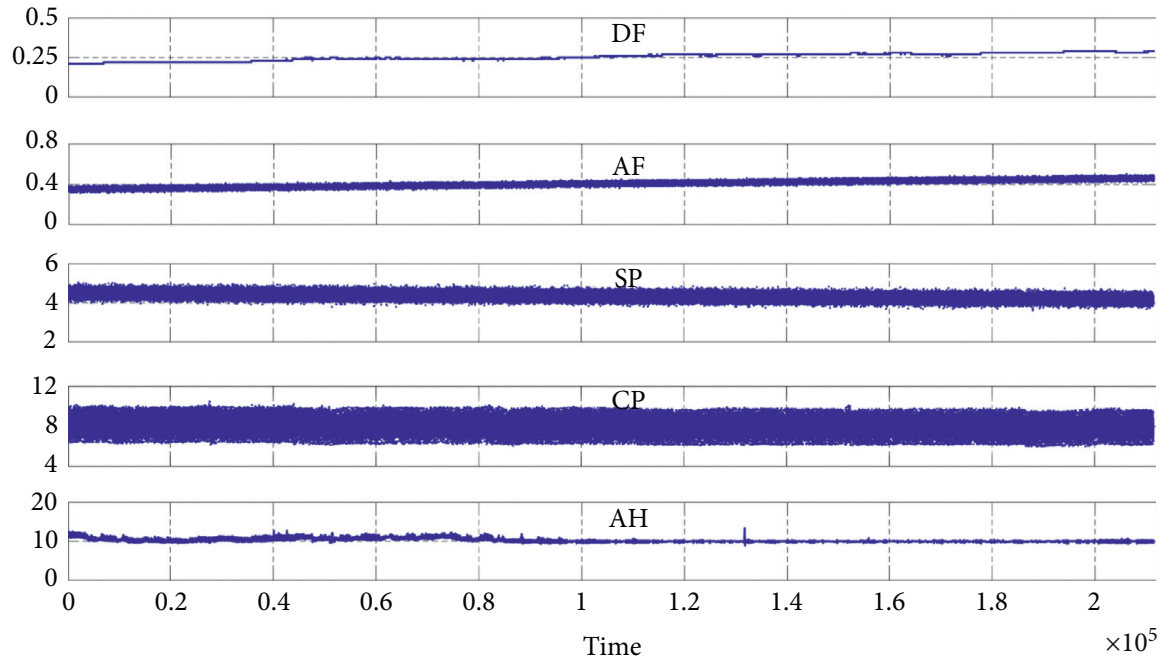

Figure 6: Monitoring data for the evaluation indexes.

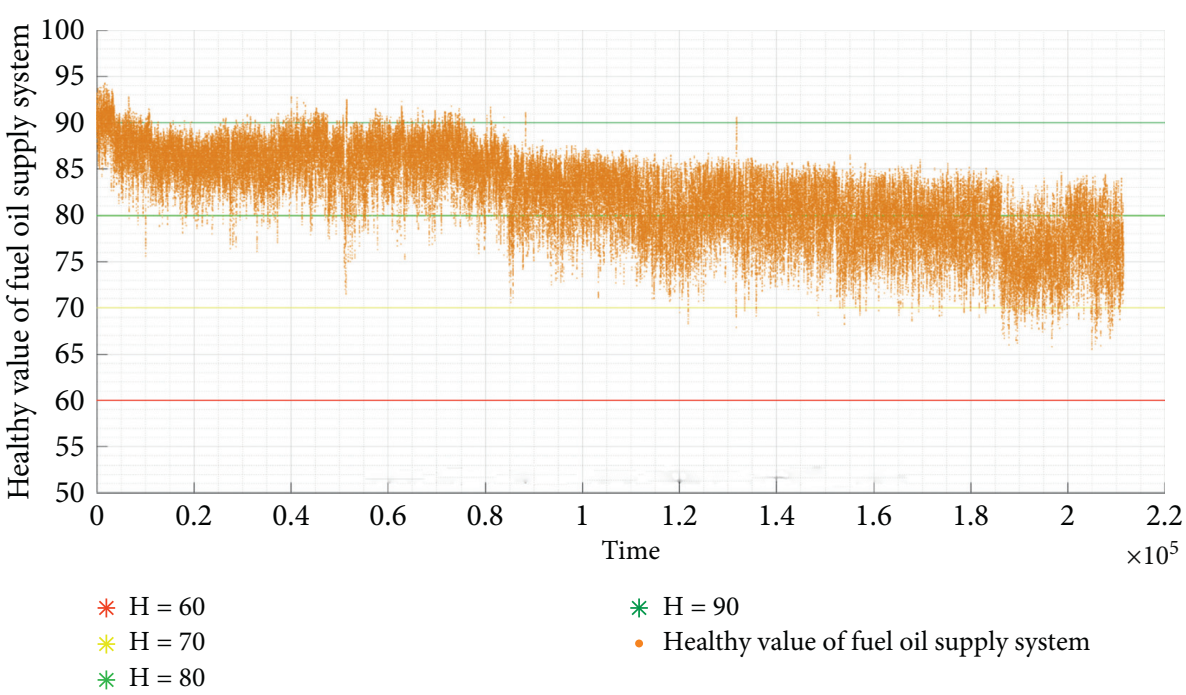

FIGURE 7: Evaluation results under long-term operation.

for the DF, AF, SP, CP, and AH downtime and abnormalities were filtered. The stable current trends are shown in Figure 6. It can be seen from the figure that the pressure difference corresponding to the DF and AF had a stable upward trend. Additionally, the pressure data corresponding to the SP and CP fluctuated in a range, and the viscosity of the index parameter of the $\mathrm{AH}$ was greatly affected by the external environment and it changed greatly over a period of time. The preprocessed data could be applied for correlation analysis and health evaluation.

The continuous calculation of the health value was achieved with the IRC method. The results are shown in Figure 7.

5.3.1. Normal Condition. The target ship was launched in 2008, and it had a life span of 11 years. Owing to performance degradation and other reasons, the actual health of the main engine oil supply system had declined to a certain level. According to Figure 7, due to performance degradation and other uncertainty reasons, the actual health of the system had declined to a certain level. The initial health value was between 90 points and 100 points, not 100 points, so the calculation results reflected the true situation. With the extension of time, the health value gradually declined, it gradually entered the 80-90 score range and 70-80 score range, and it had the trend of entering the 60-70 score range, which was about to reach the limit. The method could achieve the continuous online state monitoring of the system, and it could exhibit a potential capability in tracing the further deterioration of the system.

5.3.2. Abnormal Condition. A section of abnormal fuel viscosity data was intercepted from the real ship operation parameters to verify the response of the method to the abnormal state. As can be seen in Figure 8, there were two 


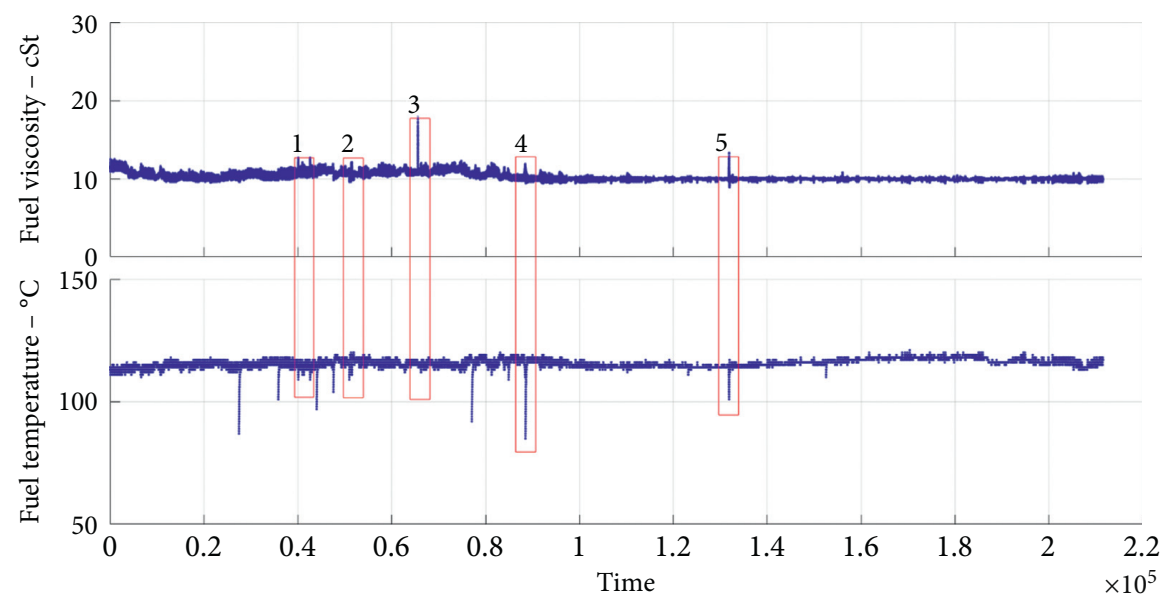

FIgURE 8: Analysis chart for abnormal fuel viscosity.

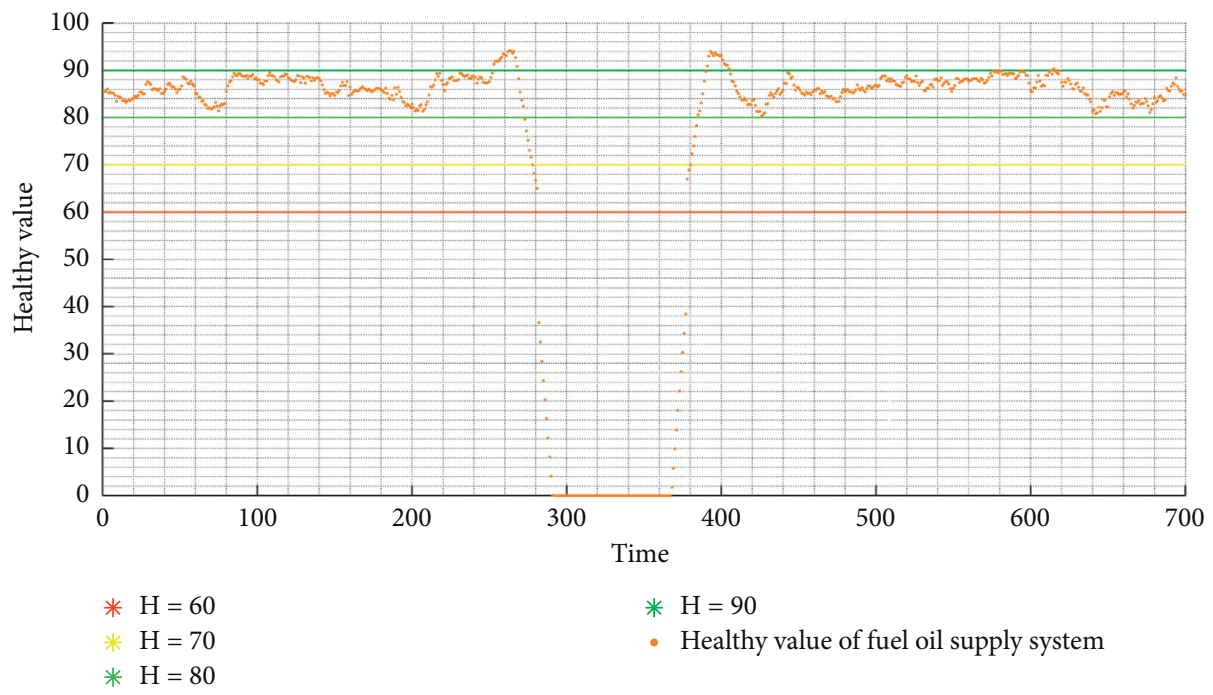

FIGURE 9: The health status assessment results under abnormal conditions.

distinct fluctuations in the fuel viscosity. In the first category, as shown in the red bar nos. 1, 2, 4, and 5, the fuel viscosity fluctuated for a short time. That is, the fluctuation appeared and disappeared rapidly. The fuel temperature also decreased in the same period. It could be judged that the fuel viscosity rose rapidly due to the decrease in the fuel temperature, and then, it returned to normal in a short time due to automatic regulation. In the second category, as shown in the red bar no. 3 , the fuel viscosity rose rapidly, exceeding the limit value.

As depicted in Figure 9, the abnormality of a single device in the system affected the overall health value of the system, resulting in the loss of system function. However, as the function of the device recovered, the health status of the system returned to normal. During this process, in the initial state, when the performance of a single device declined, the system could maintain the functionality, but the health value gradually decreased. When the performance of the device declined rapidly below a certain value, the series system lost function, and the health value was 0 points. As the device status recovered, the system health returned to normal. The change in the health value trend was consistent with the abnormal change in the fuel viscosity past the atomization heater caused by viscometer fouling. This showed that the health model proposed in this research could better reflect the health status changes under the fault condition. The health value could also be used as the health index of the system fault and the basis of CBM.

5.4. Comments and Discussion. A traditional static state evaluation is usually a one-time method for which the historical data are used to achieve the system state estimation. This method cannot achieve online monitoring, and it is not easy to control the state change of a system. The dynamic evaluation can be adjusted in real time according to the changes in the parameters, and the assessment results are 


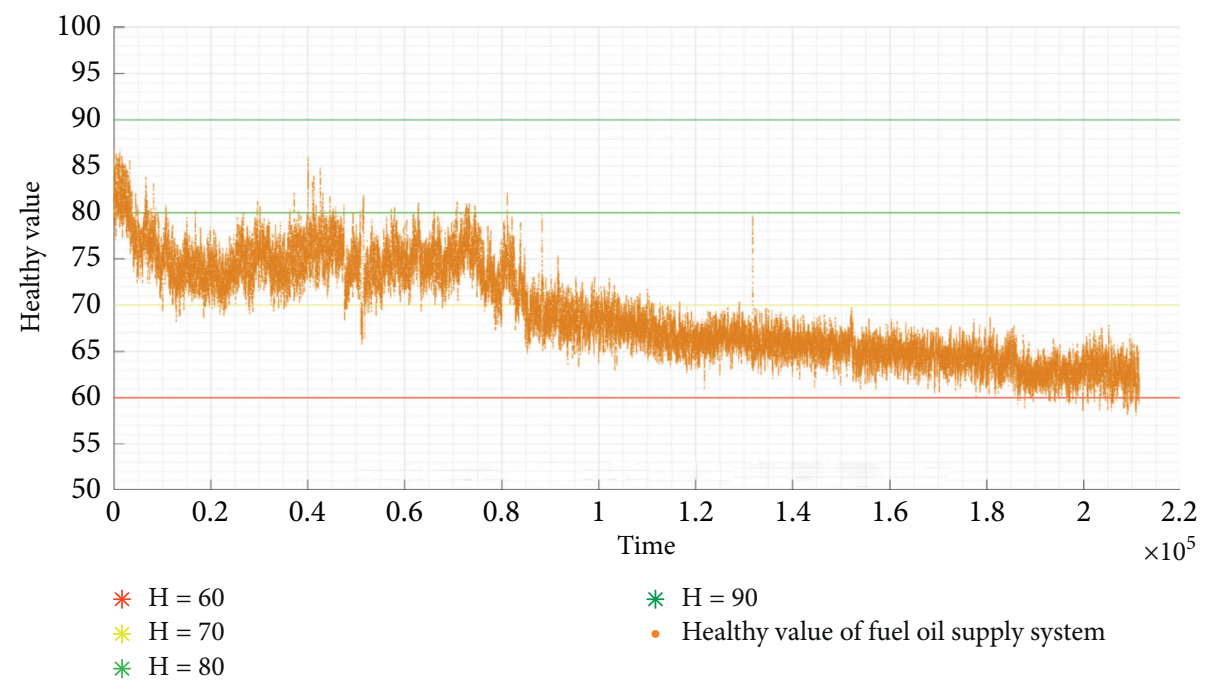

FIGURE 10: Fixed weight health status comparison.

closer to the real changes, which can provide a reference for $\mathrm{CBM}$ and auxiliary decision-making.

The proposed method provided an integral health assessment by further fusing the probability health-indicator of each component. The system status was evaluated based on multiple-related signals, thus making it possible to detect potential faults. Especially for the intelligent engine room or the unmanned ship, more attention was paid to the time when the systems and equipment could meet the function output and operation continuous. When there were problems and performance degradation for the individual equipment in the system, the effectiveness of the necessary functions of the whole system was maintained, which played an important role in making operation and maintenance decisions.

It can be seen from Figure 10 that the fixed weight system would first reach the limit (health value $=60$ ) and trigger the maintenance. The dynamic assessment system was far from the limit, and it could be used continuously, as shown in Figure 10. The system was disassembled, and it was found that the filter in the system was dirty and the performance of the system was decreased, but the output pressure and flow of the system were normal, which could meet the normal use conditions of the main engine. The dynamic evaluation results were closer to the actual state. The proposed method was more capable of capturing the dynamic degradation process of the considered system, even if it was less effective for the scenarios with low possibilities.

\section{Conclusions}

In this research, IRC was applied as a health condition assessment method for MS. Two main improvements were made in this method. One of the improvements was to apply FCST and the entropy weight as the comprehensive weight method. This method fully considered the influence of the subjective and objective factors on the weight. It also converted the comprehensive weight to an angle, it caused the proposed method to reflect the influence degree of indexes on the evaluation objects, and it had unique eigenvector and evaluation results. Another improvement was the continuous online assessment of a system that contained multiequipment and multiparameters. The health value was used as an indicator to assess the health status of the system according to the changes in the parameters. The result showed that the proposed method gave reliable and accurate assessment results that could provide a reference for CBM and decision-making.

Compared with health condition assessment methods, the IRC method had full consideration of the influence of multiparameters on a system during working conditions. The experimental results also showed that the IRC method was more effective in achieving the dynamic state assessment of a system. In future work, we plan to develop a new method based on an adaptive baseline-threshold model for real-time condition monitoring and health assessment.

\section{Data Availability}

The data used to support the findings of this study are available from the corresponding author upon request.

\section{Conflicts of Interest}

The authors declare that they have no conflicts of interest.

\section{Acknowledgments}

This project was supported by the National Key Research and Development Program of China (no. 2018YFB1601502), High-Tech Ship Scientific Research Projects (no. MC201712-C07), and the Fundamental Research Funds for the Central Universities (no. 31320190060).

\section{References}

[1] C. A. Thieme and I. B. Utne, "Safety performance monitoring of autonomous marine systems," Reliability Engineering \& System Safety, vol. 159, pp. 264-275, 2017. 
[2] S.-H. Xiong, Z.-S. Chen, J.-P. Chang, and K.-S. Chin, "On extended power average operators for decision-making: a case study in emergency response plan selection of civil aviation," Computers \& Industrial Engineering, vol. 130, pp. 258-271, 2019.

[3] Z.-S. Chen, K.-S. Chin, and K.-L. Tsui, "Constructing the geometric Bonferroni mean from the generalized Bonferroni mean with several extensions to linguistic 2-tuples for decision-making," Applied Soft Computing, vol. 78, pp. 595-613, 2019.

[4] Z.-S. Chen, C. Yu, K.-S. Chin, and L. Martínez, "An enhanced ordered weighted averaging operators generation algorithm with applications for multicriteria decision making," Applied Mathematical Modelling, vol. 71, pp. 467-490, 2019.

[5] L. Wang and N. Li, "Pythagorean fuzzy interaction power Bonferroni mean aggregation operators in multiple attribute decision making," International Journal of Intelligent Systems, vol. 35, 2020.

[6] A. Azadeh, M. Sheikhalishahi, S. M. Khalili, and M. Firoozi, "An integrated fuzzy simulation-fuzzy data envelopment analysis approach for optimum maintenance planning," International Journal of Computer Integrated Manufacturing, vol. 27, no. 2, pp. 181-199, 2014.

[7] J. Barabady and U. Kumar, "Reliability analysis of mining equipment: a case study of a crushing plant at Jajarm Bauxite Mine in Iran," Reliability Engineering \& System Safety, vol. 93, no. 4, pp. 647-653, 2008.

[8] J. Seif, A. Azadeh, M. Sheikhalishahi, and M. Yazdani, "An integrated support vector regression-imperialist competitive algorithm for reliability estimation of a shearing machine," International Journal of Computer Integrated Manufacturing, vol. 29, 2015.

[9] A. Azadeh, S. M. Asadzadeh, and J. Seif, "An integrated simulation-analysis of variance methodology for effective analysis of CBM alternatives," International Journal of Computer Integrated Manufacturing, vol. 27, no. 7, pp. 624637, 2014.

[10] M. M. Rana, W. Xiang, and E. Wang, "Smart grid state estimation and stabilisation," International Journal of Electrical Power \& Energy Systems, vol. 102, pp. 152-159, 2018.

[11] H. H. Afshari, M. Attari, R. Ahmed, A. Delbari, S. Habibi, and T. Shoa, "Reliable state of charge and state of health estimation using the smooth variable structure filter," Control Engineering Practice, vol. 77, pp. 1-14, 2018.

[12] T. Zahid, K. Xu, W. Li, C. Li, and H. Li, "State of charge estimation for electric vehicle power battery using advanced machine learning algorithm under diversified drive cycles," Energy, vol. 162, pp. 871-882, 2018.

[13] M. Xu, X. Jin, S. Kamarthi, and M. Noor-E-Alam, “A failuredependency modeling and state discretization approach for condition-based maintenance optimization of multi-component systems," Journal of Manufacturing Systems, vol. 47, pp. 141-152, 2018.

[14] A. Kumar, R. B. Chinnam, and F. Tseng, "An HMM and polynomial regression based approach for remaining useful life and health state estimation of cutting tools," Computers \& Industrial Engineering, vol. 128, pp. 1008-1014, 2019.

[15] G. S. Misyris, A. Marinopoulos, D. I. Doukas, T. Tengnér, and D. P. Labridis, "On battery state estimation algorithms for electric ship applications," Electric Power Systems Research, vol. 151, pp. 115-124, 2017.

[16] Y. Deng, H. Ying, E. Jiaqiang et al., "Feature parameter extraction and intelligent estimation of the State-of-Health of lithium-ion batteries," Energy, vol. 176, pp. 91-102, 2019.
[17] X. Wang, X. Wei, and H. Dai, "Estimation of state of health of lithium-ion batteries based on charge transfer resistance considering different temperature and state of charge," Journal of Energy Storage, vol. 21, pp. 618-631, 2019.

[18] Z. Ma, R. Yang, and Z. Wang, "A novel data-model fusion state-of-health estimation approach for lithium-ion batteries," Applied Energy, vol. 237, pp. 836-847, 2019.

[19] J. Sun, F. Wang, and S. Ning, "Aircraft air conditioning system health state estimation and prediction for predictive maintenance," Chinese Journal of Aeronautics, vol. 33, no. 3, pp. 947-955, 2020.

[20] S. Wang, L. Hou, J. Lee, and X. Bu, "Evaluating wheel loader operating conditions based on radar chart," Automation in Construction, vol. 84, pp. 42-49, 2017.

[21] S. Li, B. Su, D. L. St-Pierre, P.-C. Sui, G. Zhang, and J. Xiao, "Decision-making of compressed natural gas station siting for public transportation: integration of multi-objective optimization, fuzzy evaluating, and radar charting," Energy, vol. 140, pp. 11-17, 2017.

[22] Y. L. Wang and Y. J. Li, "Comprehensive evaluation of power transmission and transformation project based on improved radar chart," Advanced Materials Research, vol. 354-355, pp. 1068-1072, 2012.

[23] L. Wang, H. Garg, and N. Li, "Pythagorean fuzzy interactive Hamacher power aggregation operators for assessment of express service quality with entropy weight," Soft Computing, 2020.

[24] K. Ghosh, S. K. Parui, and P. Majumder, "Learning combination weights in data fusion using Genetic Algorithms," Information Processing \& Management, vol. 51, no. 3, pp. 306-328, 2015.

[25] L. Yuan and F. Xu, "Research on the multiple combination weight based on rough set and clustering analysis-the knowledge transfer risk in IT outsourcing taken as an example," Procedia Computer Science, vol. 17, pp. 274-281, 2013.

[26] K. Xu, X. Y. Ding, H. W. Chen et al., "Multi-attribute comprehensive evaluation method for power transmission and transformation projects," Applied Mechanics and Materials, vol. 521, pp. 245-251, 2014.

[27] E. R. Ziegel, "System reliability theory: models, statistical methods, and applications," Technometrics, vol. 46, no. 4, pp. 495-496, 2004.

[28] R. Gaohua, G. Chunsheng, and C. Yalin, "Study on ability evaluation system of a technical supply element based on entropy-weight combination method," in Proceedings of the 2012 International Conference on Industrial Control and Electronics Engineering, pp. 1687-1689, Xi'an, China, August 2012. 